\title{
Variations
}

Variations

Revue internationale de théorie critique

$23 \mid 2020$

Pour une gauche érotique

\section{Présentation : des topolitiques culturelles oppositionnelles}

\section{Fabien Granjon}

\section{(2) OpenEdition}

\section{Journals}

Édition électronique

URL : http://journals.openedition.org/variations/1361

DOI : 10.4000/variations. 1361

ISSN : 1968-3960

Éditeur

Les amis de Variations

\section{Référence électronique}

Fabien Granjon, « Présentation : des topolitiques culturelles oppositionnelles », Variations [En ligne], 23 | 2020, mis en ligne le 01 septembre 2020, consulté le 25 septembre 2020. URL : http:// journals.openedition.org/variations/1361 ; DOI : https://doi.org/10.4000/variations.1361

Ce document a été généré automatiquement le 25 septembre 2020

Les ami•e•s de Variations 


\title{
Présentation : des topolitiques culturelles oppositionnelles
}

\author{
Fabien Granjon
}

«On n'est pas le produit d'un sol, on est le produit de l'action qu'on y mène »

Félix-Marcel Castan

1 Le déploiement de la conflictualité sociale va de pair avec l'expérimentation de nouvelles modalités pratiques de l'agir en commun et l'articulation de plusieurs types d'expériences et de subjectivités qui ne sont évidemment pas sans lien avec les territoires (locaux, nationaux, culturels, de vie, de travail, etc.) et les communautés solidaires qui leur sont liées; là où ces luttes se déploient et dont ils peuvent aussi être les conditions spatiales. Toutes sortes d'espace peuvent ainsi (sup)porter des conflits politiques, lesquels marquent diversement, selon leur(s) objet(s), leur intensité, leur durée, etc., les référents territoriaux sur et depuis lesquels ils se déploient. On lutte, en effet, toujours depuis, dans et même éventuellement pour des espaces et des lieux ${ }^{1}$ sociogéographiques particuliers (alter-lieux, contre-lieux - Lussault, 2017 ; hétérotopies Foucault, 1984; territoire critiques - Granjon, Denouël, 2018). Les communs oppositionnels (Nicolas-Le Strat, 2016) qui, à la fois portent et naissent de l'action collective se nourrissent d'expériences critiques territorialisées et les cultures de résistance qui en découlent adviennent « dans une proximité et dans un paysage » ${ }^{2}$.

Le modeste objectif de ce sous-dossier est de saisir, à titre d'exemples et depuis des terrains variés, la manière dont les ancrages territoriaux, les politiques du conflit et les mobilisations culturelles se mêlent dans des configurations singulières que nous nommons topolitiques culturelles oppositionnelles (TCO). Du fait de cette attention aux TCO, il convient de préciser - succinctement pour l'heure - trois choses. D'une part, expliciter ce que nous entendons par «culturel»; d'autre part, donner quelque consistance à la notion de "territoire»; enfin clarifier le néologisme «topolitique ». Commençons cette opération de spécification par le dernier point. Par l'emploi du néologisme «topolitique » nous souhaitons rendre compte de l'ancrage territorial des conflits politiques (territoires politiques/politiques des territoires). Le mot a déjà été 
employé par Jacques Derrida (2001: 57; Derrida, Stiegler, 1996: 67 et seq.), afin d'évoquer l'écart grandissant entre citoyenneté et territorialité locale - en l'espèce, sous l'effet de la technologie. Notre emploi du terme, sans être orthogonal à cet usage, désigne plutôt le répertoire de liens que peuvent entretenir le politique avec la territorialité (l'inscription dans un territoire et son intégrité) et la territorialisation (le faire territoire) $)^{3}$. Il s'agit d'envisager les formes d'engagement politique territorialisées dont, au surplus, les ressorts s'avèrent culturels. Ce que nous qualifions de "culturel » relève à la fois, du sens anthropologique : la production de normes, de règles et de valeurs définissant des manières d'être et de faire (et, en l'occurrence, de justifier/ mener des luttes); et du sens sociologique, s'agissant des usages sociaux de certaines formes de connaissances, de savoirs et des arts qui, eux aussi, peuvent légitimer et/ou fixer des manières de résister et de s'opposer. Quant au territoire, il est envisagé comme le socle spatial de processus conflictuels et, dans le même mouvement, un espace saisi et singularisé par ces conflits. Les TCO décrivent ainsi des processus agonistiques ouvrant des dynamiques oppositionnelles (Negt, 2007) portés par une territorialité (un milieu composé de ressources culturelles cadrant des relations et du politique), mais produisant également de la territorialisation, c'est-à-dire faisant exister, par l'engagement de ses sujets dans diverses actions collectives et par la production de communs oppositionnels (i.e. de topiques culturels alternatifs), un espace local culturellement singulier (une localité oppositionnelle).

Quatre textes composent ce sous-dossier. Le dialogue complice entre Bernard Lubat et Fabien Granjon porte sur l'imaginaire territorialisé des luttes culturelles que mène l'artiste depuis son village natal (Uzeste), lesquelles dessinent un front culturel de résistance populaire (Granjon, 2016) et font exister, depuis plus de quarante ans, un espace oppositionnel singulier, couplant traditions rurales et avant-gardes artistiques : la Jazzcogne (une Gascogne enjazzée éprise d'émancipation). La contribution d'Alain Mélo, nous fait traverser la France et passer du Sud-Gironde au Haut-Jura. Il revient, en historien, sur les activités de La fraternelle et de la Maison du peuple de Saint-Claude, haut-lieu, depuis le XIXe siècle, du coopérativisme et d'un développement politique des pratiques culturelles en direction des ouvrier.e.s. Il montre comment ces dernier.e.s s'emparèrent de la proposition culturelle comme d'une arme politique, à la manière dont ils/elles s'étaient également saisis localement de leur consommation alimentaire et de leur protection sociale, et rend compte de l'évolution contemporaine de cette singularité. Autre temps, autre continent: l'entretien que Jacques Guyot et Danièle Téphany ont conduit avec Leo Casas Ballón - poète, musicien et écrivain péruvien -, révèle la manière dont le chant, la danse, les récits et les rituels traditionnels de la culture quechua constituent la matière-même d'une résistance à l'oppression. Pour autant, le travail de préservation de cette culture minoritaire ne se ferme ni aux processus de créolisation, ni à l'usage des technologies médiatiques d'information et de communication. De technologie il est aussi question dans le texte de Natalia Calderón Beltrán qui nous maintient dans l'hémisphère Sud. La jeune chercheuse francocolombienne éclaire les processus de technocontestation propres à Cuba, dans un contexte territorial et politique notamment marqué par l'isolement (Blocus) et la transculturation. Le détournement imprévu du technosystème et l'entretien rusé des machines esquissent une forme locale de hacking dont les Cubain.e.s semblent s'être fait une spécialité.

4 L'arpentage de ces différents territoires, dans leur couplage à des logiques politiques et culturelles en chaque cas singulières souligne la diversité des TCO. Il s'agit donc de 
l'esquisse d'un projet plus vaste consistant, depuis l'établissement d'une jurisprudence étoffée, à répondre à une série de questions ${ }^{4}$ qui nous permettraient de repérer et de qualifier la variété des manières dont se dialectisent territorialisation des agirs politiques et politisation des espaces culturels. Cet effort de recensement autoriserait également à repérer certaines des caractéristiques communes des $T C O$, au-delà de ce qui permet d'en faire une catégorie d'objets de recherche repérables. Documenter, qualifier et discuter ces déploiements de la conflictualité territoriale, sociale et culturelle ouvre à la constitution d'un espace de recherche critique, international et interdisciplinaire auquel Variations ne manquera pas de donner quelque visibilité. Rendez-vous est pris.

\section{BIBLIOGRAPHIE}

Derrida (Jacques), L'Université sans condition, Paris, Galilée, 2001.

Derrida (Jacques), Stiegler (Bernard), Échographies de la télévision, Paris, Galilée, 1996.

Di Méo (Guy), "Le renouvellement des fêtes et des festivals, ses implications géographiques ", Annales de géographie, $\mathrm{n}^{\circ}$ 643, 2005, pp. 227-243.

Foucault (Michel), « Des espaces autres », in Dits et écrits II - 1976-1988, Paris, Gallimard, 1984, pp. 1571-1581.

Granjon (Fabien), Denouël (Julie), « Avant-propos. Hétérotopies, Uz-topies et sociologie à vivre », in Denouël (Julie), Granjon (Fabien) dir., Politiques d'UZ : vivacités critiques du réel, Éditions du commun, Rennes, 2018, pp. 15-43.

Granjon (Fabien), Les UZ-topies de Bernard Lubat (Dialogiques), Paris, Outre Mesure, 2016.

Lacoste (Yves), « La géographie, la géopolitique et le raisonnement géographique », Hérodote, $\mathrm{n}^{\circ} 130,2008$, pp. 17-42.

Lussault (Michel), Hyper-lieux. Les nouvelles géographies de la mondialisation, Paris, Seuil, 2017.

Negt (Oskar), L'espace public oppositionnel, Paris, Payot, 2007.

Nicolas-Le Strat (Pascal), La travail du commun, Rennes, Éditions du commun, 2016.

\section{NOTES}

1. «Ce que le lieu contient et (r)assemble contribue à le configurer et à le faire exister comme un espace humain spécifique qu'on peut distinguer des autres, un espace doté de qualités propres, un je-ne-sais-quoi et un presque-rien qui le qualifient et le rendent reconnaissable entre tous ; symétriquement, en quelque sorte, les formes de vie sociale et les pratiques qu'un lieu autorise sont incontestablement imprégnées de ses caractères particuliers. Il devient alors impossible de séparer le lieu de ce qui d'y déroule, s'y vit, s'y trame et qui permet au lieu de ne pas être qu'un simple réceptacle... » (Lussault, $2017:$ 41-42). 
2. Guy Di Méo note par exemple que « Dans la plupart des pays du monde, notamment en Europe, au fur et à mesure que progressent la mondialisation et la décentralisation territoriale des pouvoirs, les événements festifs, neufs ou rénovés, deviennent [...] des outils localisés, franchement politiques. Les messages idéologiques qu'ils transmettent au travers de leurs formes spatiales et au rythme de leurs séquences temporelles, puisent leur contenu dans l'action et dans le projet politiques » (2005 : 231).

3. Le topolitique diffère $d u$ géopolitique (Rudolf Kjellén) dans la mesure où, s'il traite effectivement de conflits territorialisés, ceux-ci ne sont pas nécessairement des rivalités de pouvoirs institués/institutionnalisés (e.g. entre États), portant sur le gouvernement/contrôle des territoires. La dimension stratégique n'est, là, pas centrale (Lacoste, 2008).

4. De quel(s) territoire(s) la lutte sociale est-elle l'expression? De quel(s) conflit(s), le territoire est-il le contexte et le précipité ? Quelles dialectiques entre luttes politiques « from below » et production des territoires? Comment les mobilisations culturelles affectent-elles le territoire? Etc.

\section{AUTEUR}

\section{FABIEN GRANJON}

Sociologue, professeur en Sciences de l'éducation à l'Université Paris 8, membre du laboratoire Experice. 